\section{Assessing quality of life in schizophrenia}

Reading Wilkinson et al (2000), I felt that their new questionnaire tends to measure symptoms rather than quality of life. Obviously the symptoms and side-effects scale measures symptoms but a number of items in the other two scales measure symptoms as well, for example, "I lack the energy to do things". The relationship between symptoms and quality of life is complex but issues such as the availability of money or quality of accommodation must have something to do with it and neither is covered in their questionnaire.

The authors state that in measuring quality of life the measure has to be subjective, which makes sense, but whether it has to be self-reported is questionable. The authors suggested greater honesty might be outweighed by the disadvantage that no help is available if there is confusion regarding an item. I can see no advantages in using this new tool over existing tools, such as the Manchester Short Assessment of Quality of Life (Priebe et al, 1999), which is similarly short and useful in clinical practice.

Priebe, S., Huxley, P., Knight, S., et al (1999)

Application and results of the Manchester Short

Assessment of Quality of Life (MANSA). International Journal of Social Psychiatry, 45, 7-12.

Wilkinson, G., Hesdon, B., Wild, D., et al (2000) Selfreport quality of life measure for people with schizophrenia: the SQLS. British Journal of Psychiatry, 177, 42-46.

M. P. Klijnsma Mid Essex Community \& Mental Health Trust, The Linden Centre, Woodlands Way, Chelmsford CMI 7LF

Authors' reply: The Schizophrenia Quality of Life Scale (SQLS) is a measure of quality of life based on statements made by people with schizophrenia. We asked participants to state how their quality of life was affected by their mental health and the result was a mixture of symptoms, side-effects and psychosocial issues. Naturally, there is an overlap in the use of these terms, but our method elicits responses related to quality of life from a patient's perspective.

We believe that nobody can know their quality of life better than the person him- or herself. We have no reason to believe, on the basis of our findings, that people with schizophrenia are incapable of accurately representing their quality of life using the SQLS. It is of interest that we found that the people we interviewed voiced a variety of concerns, albeit not about possible financial and accommodation problems, which are, in any event, objective issues.

The measurement of quality of life is in the scientific domain and different methods, whether by questionnaire or interview, need to demonstrate reliability and validity. Thereafter, the choice of instrument requires a trade-off, representing a decision about the best instrument for a particular purpose. Factors such as ease of use, acceptability and cost must be considered.

In contrast to measures such as the Manchester Short Assessment of Quality of Life (MANSA), the SQLS has been specifically developed from interviews with people with schizophrenia, as opposed to being based on other measures. It was also developed as a measure for use in clinical trials and other research studies, as opposed to the assessment of community programmes, which is the case with the Lancashire Quality of Life Profile (Oliver et al, 1996), the measure from which the MANSA was derived. We would emphasise that the administration procedures contained in The User Manual for the SQLS (available from Oxford Outcomes) recommend, among other things, that the SQLS is completed in the presence of the researcher or is administered as an interview if the patient has difficulty with self-completion.

The SQLS has been very well received by colleagues both in the UK and internationally and the development of the instrument is continuing. It is clear to us from the response so far that the SQLS is recognised to fulfil a significant clinical and research need in relation to the assessment of quality of life in people with schizophrenia.

Oliver, J. P. J., Huxley, P. J., Bridges, K., et al (1996) Quality of Life and Mental Health Services. London: Routledge

D. Wild Oxford Outcomes, Bury Knowle Coach House, North Place, Old High Street, Headington OX3 9HY

G.Wilkinson University Department of Psychiatry, Royal Liverpool University Hospital, Liverpool

\section{Is couple therapy better than antidepressant drugs?}

The conclusion that couple therapy "is much more acceptable than antidepressant drugs" is not based on the findings of this study (Leff et al, 2000). To generalise results of a trial on desipramine, a tricyclic antidepressant (followed by trazodone and fluvoxamine), to antidepressant drugs as a whole is misleading. Other antidepressant drugs may be more acceptable than the trial drugs and the conclusions should have been limited to the drugs used.

Intention-to-treat analysis can sometimes be taken to the extreme. In this study, the majority of subjects in the antidepressant group $(56 \%)$ did not receive the antidepressant as randomised and therefore it is not surprising that the antidepressant group did poorly. It would have been interesting to see, in addition, the results of an explanatory analysis that would have shown the outcomes for those who actually received antidepressant drugs compared with those who actually received couple therapy. Although the study would, technically, no longer be a randomised trial, this would not be a problem because the baseline comparison shows that the two groups are comparable, the essence of randomisation. It is not always the case that intention-to-treat analysis is better than explanatory analysis.

Although comparatively new, the number needed to treat is now widely understood as a simple and meaningful analysis of trials. What is the improvement rate attributable to couple therapy in this trial and how many patients will need to be treated for one more patient to gain improvement with couple therapy over desipramine?

Finally, the effect of an important confounding variable has not been discussed. The results may have been confounded by marital discord that will respond better to couple therapy than to antidepressants.

These must be considered before recommending couple therapy over antidepressant drugs in people with depression living with partners.

Leff, J., Vearnals, S., Brewin, C. R., et al (2000) The London Depression Intervention Trial. Randomised controlled trial of antidepressants $v$. couple therapy in the treatment and maintenance of people with depression living with a partner: clinical outcome and costs. British Journal of Psychiatry, 177, 95-100.

L. O. Ogundipe Lyme Brook Mental Health Centre, North Staffordshire Combined NHS Trust, Bradwell Hospital, Talke Road, Newcastle-underLyme, Staffordshire ST5 7TL

Authors' reply: Dr Ogundipe is, of course, quite right in pointing out that our conclusion about the greater acceptability of couple therapy compared with antidepressant drugs can only apply to the particular 